\title{
Performing Central Neck Dissection in Patients with Papillary Thyroid Carcinoma with Clinically Node Negative, Benefits and Drawbacks: A Comparative Study
}

\author{
Ibtsam Shehta Harera ${ }^{1}$, Gamal Osman ${ }^{1}$, Rehab Hemeda ${ }^{2}$, Shady Emad Shaker ${ }^{3}$, \\ Mohamed Abdallah Zaitoun ${ }^{1}$ \\ ${ }^{1}$ General Surgery Department, Faculty of Medicine, Zagazig University, Zagazig, Egypt \\ ${ }^{2}$ Clinical Oncology and Nuclear Medicine Department, Zagazig University, Zagazig, Egypt \\ ${ }^{3}$ Internal Medicine Department, Faculty of Medicine, Zagazig University, Zagazig, Egypt
}

Email address:

Ibtsam_harera@gmail.com (I. S. Harera)

To cite this article:

Ibtsam Shehta Harera, Gamal Osman, Rehab Hemeda, Shady Emad Shaker, Mohamed Abdallah Zaitoun. Performing Central Neck Dissection in Patients with Papillary Thyroid Carcinoma with Clinically Node Negative, Benefits and Drawbacks: A Comparative Study. Journal of Surgery. Vol. 8, No. 3, 2020, pp. 90-96. doi: 10.11648/j.js.20200803.13

Received: April 28, 2020; Accepted: May 20, 2020; Published: June 4, 2020

\begin{abstract}
Context: central neck dissection in addition to total thyroidectomy as a management procedure of patients with papillary carcinoma of the thyroid [PTC] was previously found to decrease the incidence of lymph node recurrence and allow and accurate PTC surgical staging that allow making a better decision regarding adjuvant radioactive iodine (RAI) ablation. But recently, central neck dissection in patients with clinical and radiological evidences of negative cervical lymph nodes was found to have many post-operative complications. Aim: to assess benefits, complications and drawbacks of performing central neck dissection by in PTC patients. Patients and methods: This is a prospective randomized study which included 70 PTC patients who was subjected to total thyroidectomy with or without neck dissection; all included patients have clinically negative cervical lymph nodes, $40(60 \%)$ underwent total thyroidectomy in addition to central neck dissection and $30(40 \%)$ patients were subjected to only total thyroidectomy without central neck dissection. We followed our patients for five years to detect recurrence, RFS and OS rates. Results: We found that operative time was longer in patients underwent central block dissection $(\mathrm{p}=0.049)$. Recurrence free survival and overall survival rates were not significantly different among both included groups, which denoted that central block neck dissection has no survival benefits in PTC patients. Conclusions: We have concluded that performing central neck dissection in PTC with clinically and radiologically negative lymph nodes has no benefits in increasing patients' survival or decreasing recurrence rate and might lead to prolongation of operation time, increasing post-operative morbidity.
\end{abstract}

Keywords: Central Neck Dissection, Papillary Thyroid Carcinoma, Recurrence, Survival

\section{Introduction}

Recently differentiated thyroid carcinoma has experienced a sharp elevation in its rate all over the world, and it has become the most prevalent malignancy [1]. Papillary carcinoma of the thyroid gland [PTC] forms about $90 \%$ of all malignant thyroid tumors and it still has a favorable prognosis and better patients' survival rates [2]. But PTC has a higher incidence of spread to cervical lymph nodes that could be related to higher incidence of local recurrence and distant metastasis, which negatively affects the survival of patients [3-6].

Performing central neck dissection in addition to thyroidectomy as a prophylactic surgical management of PTC was previously found to decrease the incidence of lymph node recurrence and allow and accurate PTC surgical staging that allow making a better decision regarding adjuvant radioactive iodine (RAI) ablation [3, 7-9]. But recently, central neck dissection in clinically node negative was found to have many post-operative complications with uncertain evidences of improving PTC patients' survival or decreasing its recurrence [3, 10-13]. So, the benefits of 
central neck dissection remain a controversial issue in PTC patients with clinically node-negative.

In the current study we aimed to assess benefits, complications and drawbacks of performing central neck dissection by comparing 2 cohorts of PTC patients the first cohort underwent total thyroidectomy with central neck dissection while the second cohort underwent total thyroidectomy without central neck dissection.

\section{Materials and Methods}

This is a prospective randomized study which included 70 PTC patients who underwent total thyroidectomy with or without central neck dissection through conventional transcervical approach; all included patients have clinically negative cervical lymph nodes from October 2014 to November 2019 in department of General surgery, Faculty of Medicine Zagazig University hospitals. Of included 70 PTC patients, $40(60 \%)$ underwent total thyroidectomy in addition to central neck dissection and $30(40 \%)$ patients underwent only total thyroidectomy without central neck dissection.

The decision of performing central neck dissection has been dependent on the status of PTC and on surgeon's opinion and on patient's acceptance. In case of performing bilateral central neck dissection we removed; pre-laryngeal, pre-tracheal in addition to para-tracheal groups of lymph nodes bilaterally, while in case of unilateral central neck dissection we removed the same lymph nodes in the ipsilateral site of PTC. We followed our patients for five years to detect recurrence, RFS and OS rates.

\subsection{Inclusion Criteria}

Patients aged from 20-50 years old, Patients accepted to be included in the study.

Operable PTC patients no extrathyroidal extention, less than $4 \mathrm{~cm}$ in size and with clinical and radiological evidence of absence of cervical lymph node metastases were included in the study.

\subsection{Exclusion Criteria}

Patients with PTC of more than $4 \mathrm{~cm}$ in size; presence of extra-thyroidal extension; pre-operative presence of clinical or radiological evidence of cervical lymph node metastasis, recurrent disease, distant metastasis or concomitant cancer were excluded from the study.

All included PTC patients gave a written consent to be included in the study after detailed explanation of the surgical procedures and aim of the study, and the study protocol was approved by "the local ethical institutional review board of Faculty of medicine, Zagazig University".

All patients underwent ultrasonography and computed tomography to evaluate the cervical lymph nodes status preoperatively. We defined clinically, node-negative state by the absence of suspicious cervical lymph nodes on ultrasonography and computed tomography images.

We performed staging of PTC according to the 7 th edition of "American Joint Committee on Cancer/International Union for Cancer Control (AJCC/UICC)". All included PTC patients underwent flexible laryngoscopy pre-operatively and after surgery. We considered persisting paralysis of the vocal cord for more than six months as a permanent paralysis. We defined permanent hypoparathyroidism when the levels of serum parathyroid hormone were less than $15 \mathrm{pg} / \mathrm{ml}$ continuously for $>6$ months. We considered the occurrence of abnormal masses during physical evaluation, neck ultrasonography, or computed tomography and confirmed by FNAC as development of recurrence.

We assessed resected lymph nodes number, occurrence of postoperative complications, the need for RAI ablation, recurrence free survival rate and overall survival rates and compared between the two included cohorts regarding these parameters.

\subsection{Surgery}

All operations were done by the same 3 surgeons so techniques of thyroidectomy and lymph node dissection techniques were similar for all included patients. Identification and exposure of the recurrent laryngeal nerves has been done till their points of insertion in the larynx, and we have identified and preserved the parathyroid glands.

We assessed levels of serum calcium and parathyroid hormones on the $1^{\text {st }}$ postoperative day, and later on according to subsequent clinical evaluation.

For statistical analysis we used "Statistical Package for the Social Sciences version 20.0 (SPSS Inc, Chicago, IL, USA)". We have used Chi-square or Fisher's exact tests to compare between categorical variables whenever appropriate, and used T-test, Mann- Whitney U-test and Wilcoxon signed rank test for comparison between continuous variables. We calculated survival rates using the Kaplan-Meier survival curves and considered $\mathrm{p}<0.05$ as statistically significant.

\section{Results}

Patients' demographics and clinic-pathological data of all included PTC patients were detailed in Table 1.

We found that size of the tumor was larger in the group of patients underwent central block neck dissection than patients underwent only thyroidectomy $(p=0.006)$, incidence of multifocal tumor $(\mathrm{p}=0.005)$, extra-thyroid extension $(\mathrm{p}=0.002)$, higher $\mathrm{T}$ stage, presence of lympho-vascular and peri-neural invasion $(\mathrm{p}=0.003)$, were higher in the group of patients underwent central block neck dissection than patients underwent only thyroidectomy.

We found that number of removed lymph nodes in central block neck dissection group was $7 \pm 4$ (range 3-30). We found no significant correlations between both included group of patients regarding age or sex of the patients, grade of tumors or dose of radioactive iodine ablation.

Operative results

We found that operative time was longer in patients underwent central block dissection $(\mathrm{p}=0.049)$.

Postoperative findings: Table 2; Figure 1. 
Transient hypo-calcemia and recurrent laryngeal nerve injury was higher in the group of patients underwent central block dissection than in the other group underwent only thyroidectomy ( $\mathrm{p}=0.039$ and 0.029 respectively).

Recurrence and follow-up results: Table 2; Figure 2.

We assessed the recurrence of the tumor; clinically, radiologically and by assessment of serum thyroglobulin. We found no statistically significant differences in PTC recurrence between both included groups. Recurrence free survival and overall survival rates were not significantly different among both included groups, which denoted that central block neck dissection has no survival benefits in PTC patients.

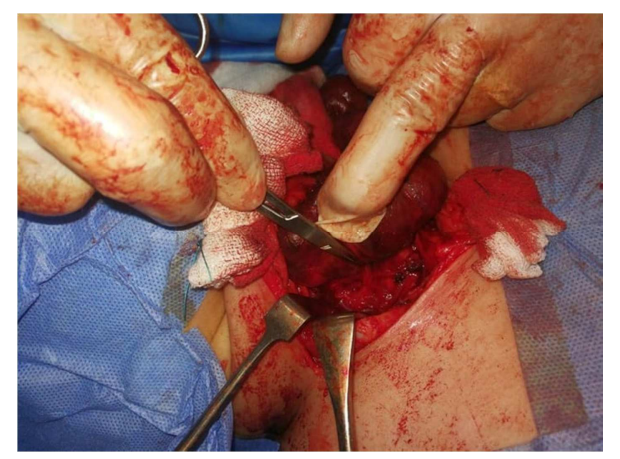

A

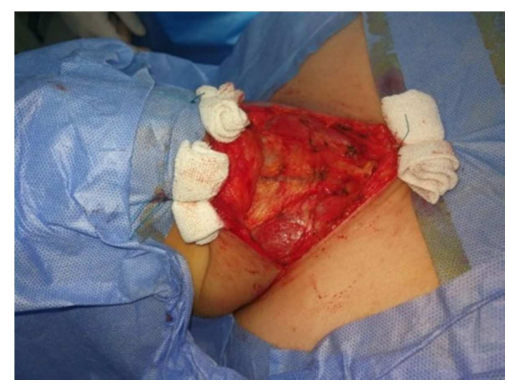

B

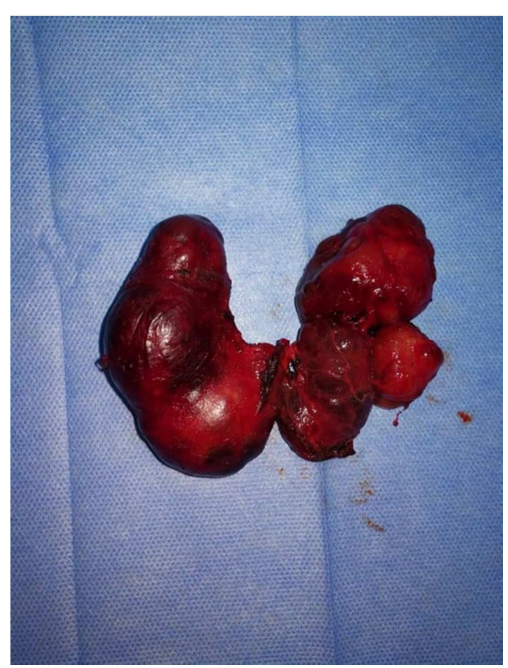

C

Figure 1. Total thyroidectomy (A and B) and post-operative appearance of the thyroid gland $(C)$.

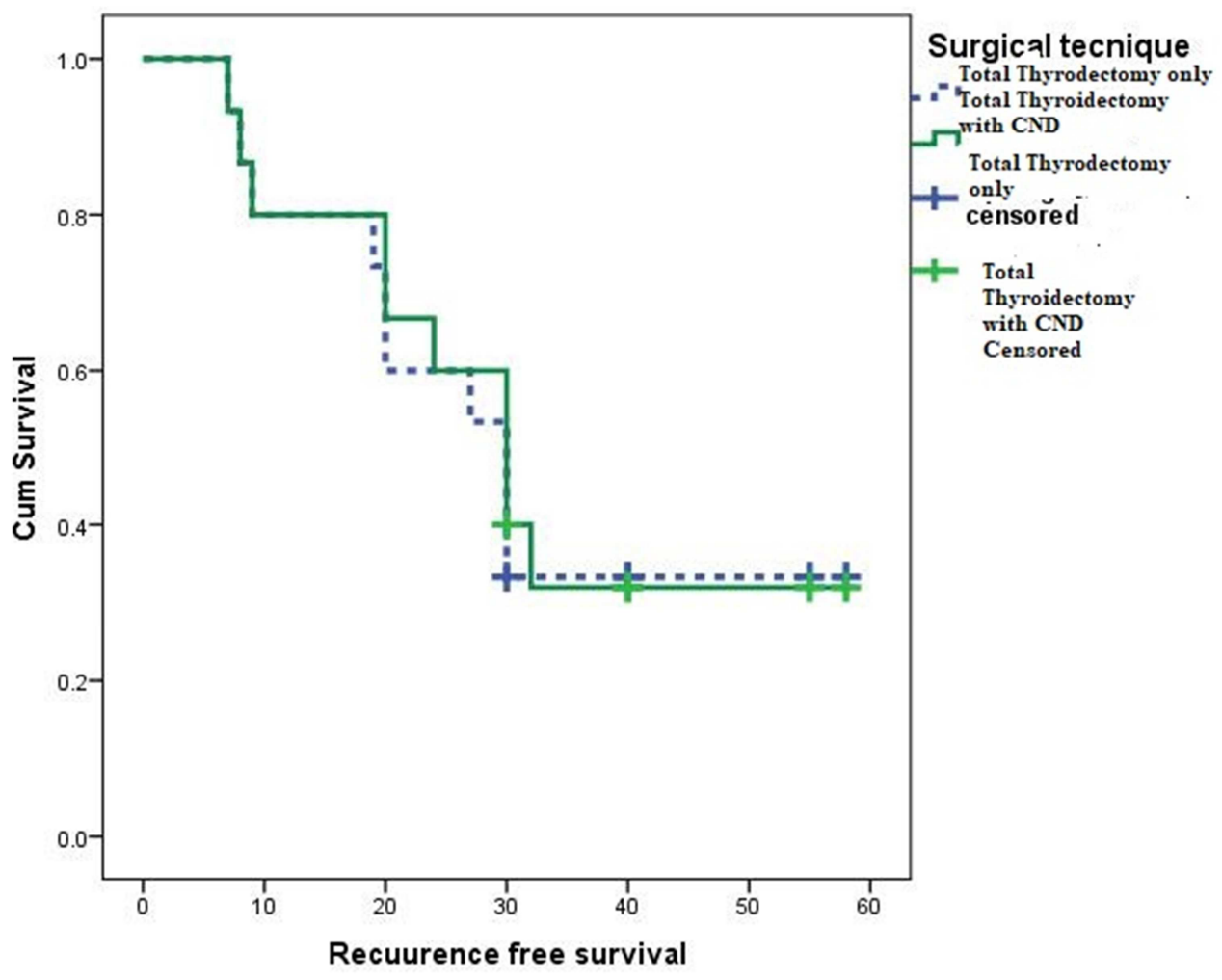




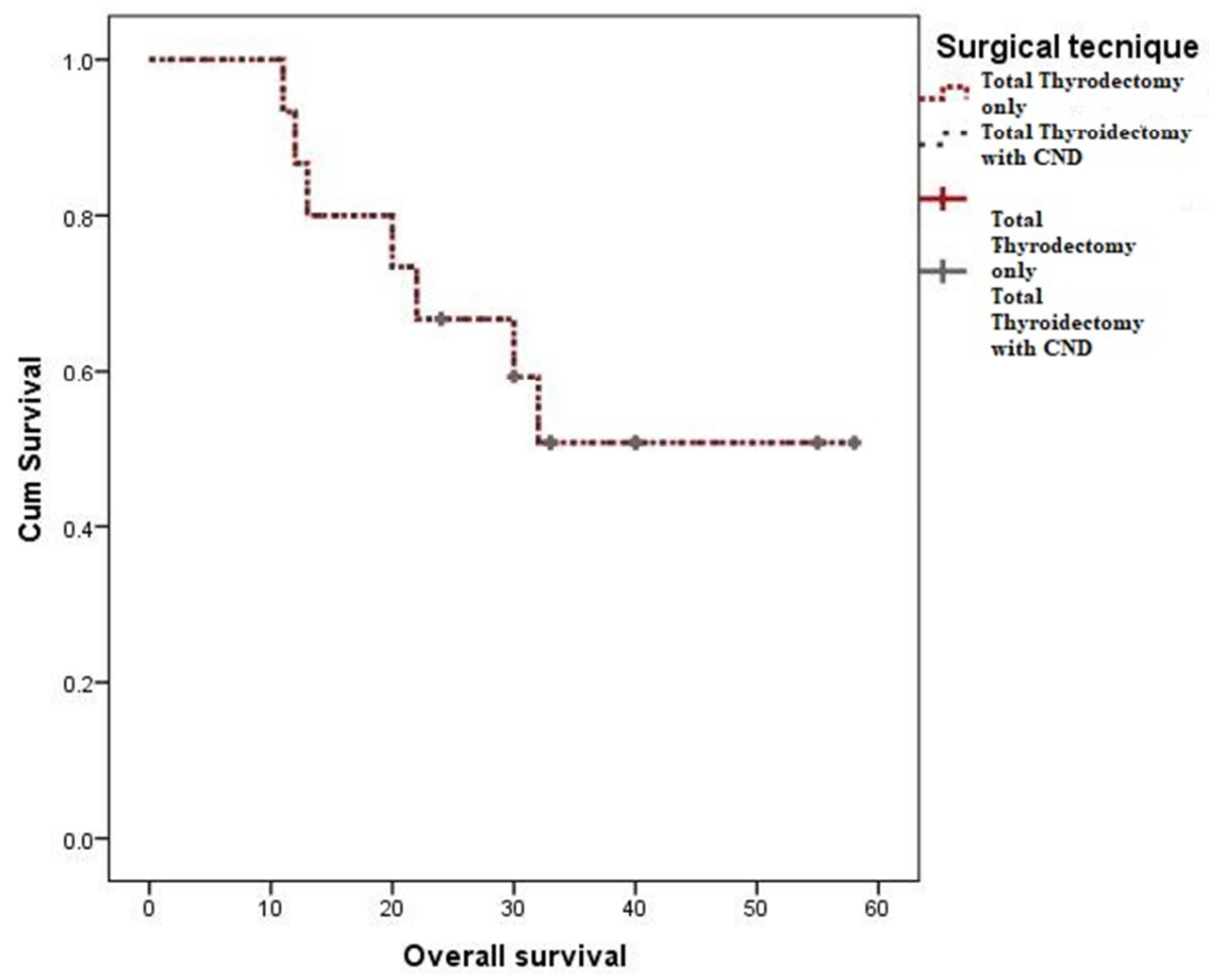

B

Figure 2. Kaplan Meier survival curves of RFS rate (A) and OS rate (B) of included PTC patients.

Table 1. Associations between both included two groups of PTC patients regarding demographic, pathological, and operative data.

\begin{tabular}{|c|c|c|c|c|}
\hline \multirow{3}{*}{ Variables } & \multirow{3}{*}{$\begin{array}{l}\text { Total } \\
\mathbf{N}=\mathbf{7 0}\end{array}$} & \multicolumn{3}{|l|}{ Surgical techniques } \\
\hline & & Total Thyroidectomy with block neck dissection & \multirow{2}{*}{$\begin{array}{l}\text { Total Thyroidectomy } \\
\mathrm{N}=\mathbf{3 0}(\mathbf{4 3} \%)\end{array}$} & \multirow{2}{*}{$\mathbf{p}$} \\
\hline & & $\mathrm{N}=40(57 \%)$ & & \\
\hline \multicolumn{5}{|l|}{ Age groups: } \\
\hline$<40$ years old & $45(64.3)$ & $25(62.5)$ & $20(66.7)$ & \multirow{2}{*}{0.099} \\
\hline$>40$ years old & $25(35.7)$ & $15(37.5)$ & $10(33.3)$ & \\
\hline \multicolumn{5}{|l|}{ Gender: } \\
\hline Female & $55(78.5)$ & $33(82.5)$ & $22(73.3)$ & \multirow{2}{*}{1} \\
\hline Male & $15(21.5)$ & $7(27.5)$ & $8(23.7)$ & \\
\hline \multicolumn{5}{|l|}{ Multifocality: } \\
\hline Present & $38(54.2)$ & $28(66.7)$ & $10(33.3)$ & \multirow{2}{*}{0.005} \\
\hline Absent & $32(45.8)$ & $12(33.3)$ & $20(66.7)$ & \\
\hline Size: & $1-4 \mathrm{~cm}$ & $2-4 \mathrm{~cm}$ & $1-2.5 \mathrm{~cm}$ & 0.006 \\
\hline \multicolumn{5}{|c|}{ Extra-capsular invasion } \\
\hline Present & $28(40)$ & $22(66.7)$ & $6(33.3)$ & \multirow{2}{*}{0.002} \\
\hline Absent & $42(60)$ & $18(33.3)$ & $24(66.7)$ & \\
\hline \multicolumn{5}{|c|}{ Histopathological subtype: } \\
\hline Classical PTC & $50(13.4)$ & $25(71.4)$ & $25(50)$ & \multirow{3}{*}{0.005} \\
\hline Follicular PTC & $15(20)$ & $12(17.4)$ & $3(50)$ & \\
\hline Other subtypes & $5(23.3)$ & $3(7.2)$ & $2(42.9)$ & \\
\hline \multicolumn{5}{|c|}{ Locoregional infiltration: } \\
\hline present & $28(40)$ & $22(66.7)$ & $6(33.3)$ & \multirow{2}{*}{0.003} \\
\hline Absent & $42(60)$ & $18(33.3)$ & $24(66.7)$ & \\
\hline
\end{tabular}

${ }^{*} \mathrm{p}<0.05$ is statistically significant $* * \mathrm{p} \leq 0.001$ is statistically highly significant. 
Table 2. Associations between both included two groups of PTC patients regarding post-operative and patient outcome.

\begin{tabular}{|c|c|c|c|c|}
\hline \multirow{3}{*}{ Variables } & \multirow{3}{*}{$\begin{array}{l}\text { Total } \\
\mathbf{N}=70(\%)\end{array}$} & \multicolumn{3}{|l|}{ Surgical techniques } \\
\hline & & Total Thyroidectomy with block neck dissection & Total Thyroidectomy & \\
\hline & & $\mathrm{N}=40(57 \%)$ & $\mathrm{N}=30(43 \%)$ & $\mathbf{p}$ \\
\hline \multicolumn{5}{|c|}{ Postoperative hypocalcaemia: } \\
\hline Absent & $60(85.7)$ & $33(82.5)$ & $27(90)$ & \multirow{2}{*}{0.039} \\
\hline Present & $10(14.3)$ & $7(17.5)$ & $3(10)$ & \\
\hline \multicolumn{5}{|c|}{ Postoperative recurrent laryngeal nerve paralysis: } \\
\hline Absent & $63(90)$ & $35(87.5)$ & $28(92)$ & \multirow{2}{*}{0.029} \\
\hline Present & $7(10)$ & $5(12.5)$ & $2(8)$ & \\
\hline \multicolumn{5}{|c|}{ Recurrence: } \\
\hline Absent & $48(68.6)$ & $26(65)$ & $22(73.3)$ & \multirow{2}{*}{0.075} \\
\hline Present & $22(31.4)$ & $14(35)$ & $8(26.7)$ & \\
\hline \multicolumn{5}{|l|}{ Death } \\
\hline No & $45(64.3)$ & $23(57.5)$ & $22(73.3)$ & \multirow{2}{*}{0.085} \\
\hline Yes & $25(35.7)$ & $17(42.5)$ & $8(26.7)$ & \\
\hline \multicolumn{5}{|c|}{ Recurrence free survival: } \\
\hline Median & 44.5 & 35 & 39 & \multirow{2}{*}{0.0 .73} \\
\hline Range & $20-60$ & $18-49$ & $20-60$ & \\
\hline \multicolumn{5}{|c|}{ Overall survival: } \\
\hline Median & 51 & 49 & 45 & \multirow{2}{*}{0.098} \\
\hline Range & $30-60$ & $29-56$ & $26-50$ & \\
\hline
\end{tabular}

${ }^{*} \mathrm{p}<0.05$ is statistically significant $* * \mathrm{p} \leq 0.001$ is statistically highly significant.

\section{Discussion}

Performing central neck dissection in PTC patients who were found to be node negative as a prophylactic procedure against long term recurrence and its survival benefits remains a controversial issue [2].

Previous researchers who advised and supported such procedure as it supposed to decrease PTC recurrence risks and the need for reoperation in addition to more accurate staging through identification of occult metastases in the cervical lymph nodes that affects taking decisions about the need for postoperative RAI ablation [7-10, 14, 15]. But many previous researchers found a contradictory results regarding central neck dissection that it did not decrease rates of tumor recurrence, did not improve patients survival in addition to more risks of causing post-operative complications as vocal cord palsy and hypo-parathyroidism [15-18].

In the current comparative prospective study we found that performing central neck dissection was of no statistically significant values regarding reduction of rates of recurrence more than total thyroidectomy alone, additionally no differences in survival benefits in patients subjected to central neck dissection more than patients subjected to only total thyroidectomy, our results were similar to results of Yoo et al., [2], Kim et al., [13] and Zetoune et al., [18] who showed that central neck dissection has no advantage regarding decreasing recurrence of the disease or improving patients out come in comparison to thyroidectomy alone. In the 2006 American Thyroid Association (ATA) guidelines of PTC management performing central neck dissection was recommended routinely for PTC patients even with nodes negative and regardless of the tumor size [19]. But in year 2009 and year 2015 the ATA guidelines limit the need for central neck dissection to advanced PTC (T3 and T4) [19, 20]. Central block neck dissection was not recommended for PTC with clinically and radiologically negative cervical lymph nodes as showed by the guidelines of "British Thyroid Association (BTA)" in case of young patients aged $<45$ years old, solitary tumor with a size less than $4 \mathrm{~cm} \mathrm{[21],} \mathrm{that} \mathrm{was} \mathrm{in}$ line with our results. Similarly the 2018 NCCN guidelines did not recommend preforming central neck dissection in case of clinically and radiologically negative lymph nodes $[22,23]$.

Conflicting results were found by $\mathrm{Su}$ et al., [24] and Calo et al., [25], Zhao et al. [8], who showed that central block neck dissection in addition to total thyroidectomy in PTC patients decreased risks of recurrence but increasing incidence of hypoparathyroidism and recurrent laryngeal nerve injury. Su et al., [24] stated that performing central neck dissection with thyroidectomy for PTC patients is a safe and efficient surgical procedure, as it not only removes occult central lymph node metastases, but also it leads to marked reduction in rate of PTC local recurrence [24]. Based on our recent report and other researches However, based on the results of the present study and results of former studies, central block neck dissection in addition to total thyroidectomy in PTC patients with clinically node-negative has no benefits regarding recurrence or survival. Su et al., [24] demonstrated different results that central neck dissection did not increase the incidence of damage to the parathyroid glands or increased the rates of recurrent laryngeal nerve injury [24]. Shen et al. revealed a marked reduction in PTC recurrence in patients underwent central neck dissection [26]. Su et al., [24] recommend performing central neck dissection in the same time of performing thyroidectomy as PTC has high incidence of cervical lymph node metastases that was in line with So et al., [27].

$\mathrm{Su}$ et al., [24] demonstrated results different from us that there was no increased morbidity and post-operative complications in patients underwent neck dissection.

However, the $\mathrm{Su}$ et al., [24], study has many limitations which make it less accurate than our current study. First, its 
retrospective non-randomized nature while our study is a prospective randomized one. Additionally in their study the decision to perform a central neck dissection was related to surgeon's preference and was not individualized for every patient.

\subsection{Strengths of Our Work}

Prospective randomized nature of the study.

Long term follow-up of patients for recurrence and survival rates which were lacked in other reports.

The comparative nature of that study which allows detection of differences between studied groups.

\subsection{Limitations of Our Work}

First, the study included a relatively small number of PTC patients. Second, most studied tumors were less than $3 \mathrm{~cm}$ in size so it was difficult to determine benefits of central neck dissection in tumors sized more than $4 \mathrm{~cm}$. Further studies that included with larger number of PTC patients and included larger tumors are needed to overcome these limitations and determine differences in recurrences between included groups.

\section{Conclusion}

We have concluded that performing central neck dissection in PTC with clinically and radiologically negative lymph nodes has no benefits in increasing patients' survival or decreasing recurrence rate and might lead to prolongation of operation time, increasing post-operative morbidity by increasing incidence of hypopathyroidism and recurrent laryngeal nerve palsy.

\section{References}

[1] Gambardella C, Patrone R, DiCapua F, Offi CH, Mauriello C, Clarizia $\mathrm{G}$ et al., The role of prophylactic central compartment lymph node dissection in elderly patients with differentiated thyroid cancer: a multicentric study BMC Surgery 2019, 18: 110.

[2] Yoo BJ, Song CM, Ji YB, Lee JY, Park HJ and Tae K. Efficacy of Central Neck Dissection for Clinically NodeNegative Papillary Thyroid Carcinoma: Propensity Scoring Matching. Front. Endocrinol. 2019; 10: 172.

[3] Mazzaferri EL, Doherty GM, Steward DL. The pros and cons of prophylactic central compartment lymph node dissection for papillary thyroid carcinoma. Thyroid. 2009; 19: 683-9.

[4] Zaydfudim V, Feurer ID, Griffin MR, Phay JE. The impact of lymph node involvement on survival in patients with papillary and follicular thyroid carcinoma. Surgery. 2008; 144: 1070-8.

[5] Podnos YD, Smith D, Wagman LD, Ellenhorn JD. The implication of lymph node metastasis on survival in patients with well-differentiated thyroid cancer. Am Surg. 2005; 71: 731-4.

[6] Zuniga S, Sanabria A. Prophylactic central neck dissection in stage N0 papillary thyroid carcinoma. Arch Otolaryngol Head Neck Surg. 2009; 135: 1087-91.
[7] Zhao WJ, Luo H, Zhou YM, Dai WY, Zhu JQ. Evaluating the effectiveness of prophylactic central neck dissection with total thyroidectomy for cN0 papillary thyroid carcinoma: An update meta-analysis. Eur J Surg Oncol. (2017a) 43: 19892000.

[8] Zhao W, You L, Hou X, Chen S, Ren X, Chen G, et al. The effect of prophylactic central neck dissection on locoregional recurrence in papillary thyroid cancer after total thyroidectomy: a systematic review and metaanalysis. Ann Surg Oncol. (2017b) 24: 2189-98.

[9] Popadich A, Levin O, Lee JC, Smooke-Praw S, Ro K, Fazel $\mathrm{M}$, et al. A multicenter cohort study of total thyroidectomy and routine central lymph node dissection for $\mathrm{cN} 0$ papillary thyroid cancer. Surgery. (2011) 150: 1048-57.

[10] Garcia A, Palmer BJ, Parks NA, Liu TH. Routine prophylactic central neck dissection for low-risk papillary thyroid cancer is not cost-effective. Clin Endocrinol. (2014) 81: 754-61.

[11] Giordano D, Valcavi R, Thompson GB, Pedroni C, Renna L, Gradoni P, et al. Complications of central neck dissection in patients with papillary thyroid carcinoma: results of a study on 1087 patients and review of the literature. Thyroid. (2012) 22: 911-7.

[12] Chisholm EJ, Kulinskaya E, Tolley NS. Systematic review and meta-analysis of the adverse effects of thyroidectomy combined with central neck dissection as compared with thyroidectomy alone. Laryngoscope. (2009) 119: 1135-9.

[13] Kim SK, Woo JW, Lee JH, Park I, Choe JH, Kim JH, et al. Prophylactic central neck dissection might not be necessary in papillary thyroid carcinoma: analysis of 11,569 cases from a single institution. J Am Coll Surg. (2016) 222: 853-64.

[14] Zheng CM, Ji YB, Song CM, Ge MH, Tae K. Number of metastatic lymph nodes and ratio of metastatic lymph nodes to total number ofretrieved lymph nodes are risk factors for recurrence in patients with clinically node negative papillary thyroid carcinoma. Clin ExpOtorhinolaryngol. (2018) 11: 58.

[15] Calò PG, Lombardi CP, Podda F, Sessa L, Santini L, Conzo G. Role of prophylactic central neck dissection in clinically nodenegative differentiated thyroid cancer: assessment of the risk of regional recurrence. Updates Surg. (2017) 69: 241-8.

[16] Raffaelli M, De Crea C, Sessa L, Giustacchini P, Revelli L, Bellantone $\mathrm{C}$, et al. Prospective evaluation of total thyroidectomy versus ipsilateral versus bilateral central neck dissection in patients with clinically node-negative papillary thyroid carcinoma. Surgery. (2012) 152: 957-64.

[17] Ji YB, Song CM, Sung ES, Jeong JH, Lee CB, Tae K. Postoperative hypoparathyroidism and the viability of the parathyroid glands during thyroidectomy. Clin Exp Otorhinolaryngol. (2017) 10: 265-71.

[18] Zetoune T, Keutgen X, Buitrago D, Aldailami H, Shao H, Mazumdar M, et al. Prophylactic central neck dissection and local recurrence in papillary thyroid cancer: a meta-analysis. Ann Surg Oncol. (2010) 17: 3287-93.

[19] Cooper DS, Doherty GM, Haugen BR, Kloos RT, Lee SL, Mandel SJ, et al. Revised American Thyroid Association management guidelines for patients with thyroid nodules and differentiated thyroid cancer: the American Thyroid Association (ATA) guidelines taskforce on thyroid nodules and differentiated thyroid cancer. Thyroid. (2009) 19: 1167214. 
[20] Haugen BR, Alexander EK, Bible KC, Doherty GM, Mandel SJ, Nikiforov YE, et al. 2015 American Thyroid Association management guidelines for adult patients with thyroid nodules and differentiated thyroid cancer: the American thyroid association guidelines task force on thyroid nodules and differentiated thyroid cancer. Thyroid. (2016) 6: 1-133.

[21] Perros P, Boelaert K, Colley S, Evans C, Evans RM, Gerrard $\mathrm{Ba} \mathrm{G}$, et al. Guidelines for the management of thyroid cancer. Clin Endocrinol. (2014) 81: 1-122.

[22] Viola D, Materazzi G, Valerio L, Molinaro E, Agate L, Faviana P, et al. Prophylactic central compartment lymph node dissection in papillary thyroid carcinoma: clinical implications derived from the first prospective randomized controlled single institution study. J Clin Endocrinol Metab. (2015) 100: 1316-24.

[23] Network NCC. NCCN Clinical Practice Guidelines in Oncology. Thyroid Carcinoma V. 12018 (2018).
[24] $\mathrm{Su} \mathrm{H}, \mathrm{Li}$ Y. Prophylactic central neck dissection and local recurrence in papillary thyroid microcarcinoma: a metaanalysis. Braz. J. Otorhinolaryngol. 2019; 85: 237-43.

[25] Calo PG, Conzo G, Raffaelli M, Medas F, Gambardella C, De Crea $\mathrm{C}$, et al. Total thyroidectomy alone versus ipsilateral versus bilateral prophylactic central neck dissection in clinically node-negative differentiated thyroid carcinoma. A retrospective multicenter study. Eur J Surg Oncol. (2017) 43: $126-32$.

[26] Shen WT, Ogawa L, Ruan D, Suh I, Duh QY, Clark OH. Central neck lymph node dissection for papillary thyroid cancer: the reliability of surgeon judgment in predicting which patients will benefit. Surgery. 2010; 148: 398-403.

[27] So YK, Son YI, Hong SD, Seo MY, Baek CH, Jeong HS, et al. Subclinical lymph node metastasis in papillary thyroid microcarcinoma: a study of 551 resections. Surgery. 2010; 148: 526-31. 